\title{
openheart Genome-wide association study-based prediction of atrial fibrillation using artificial intelligence
}

\author{
Oh-Seok Kwon, ${ }^{1}$ Myunghee Hong, ${ }^{1}$ Tae-Hoon Kim, ${ }^{1}$ Inseok Hwang, ${ }^{1}$ \\ Jaemin Shim, ${ }^{2}$ Eue-Keun Choi, ${ }^{3}$ Hong Euy Lim, ${ }^{4}$ Hee Tae Yu, ${ }^{1}$ Jae-Sun Uhm, ${ }^{1}$ \\ Boyoung Joung, ${ }^{1}$ Seil Oh, ${ }^{3}$ Moon-Hyoung Lee, ${ }^{1}$ Young-Hoon Kim, ${ }^{2}$ \\ Hui-Nam Pak (D) ${ }^{1}$
}

- Additional supplemental material is published online only. To view, please visit the journal online (http://dx.doi.org/10. 1136/openhrt-2021-001898)

To cite: Kwon 0-S, Hong M, Kim T-H, et al. Genome-wide association study-based prediction of atrial fibrillation using artificial intelligence. Open Heart 2022;9:e001898.

doi:10.1136/

openhrt-2021-001898

Received 8 November 2021 Accepted 5 January 2022
Check for updates

(C) Author(s) (or their employer(s)) 2022. Re-use permitted under CC BY. Published by BMJ.

${ }^{1}$ Cardiology, Yonsei University Health System, Seodaemun-gu, Seoul, Korea (the Republic of) ${ }^{2}$ Cardiovascular Center, Korea University Medical Center, Seoul, Korea (the Republic of) ${ }^{3}$ Cardiology, Seoul National University, Seoul, Korea (the Republic of

${ }^{4}$ Cardiology, Hallym University Sacred Heart Hospital, Anyang, Gyeonggi-do, Korea (the Republic of)

Correspondence to Dr Hui-Nam Pak; hnpak@ yuhs.ac

\section{ABSTRACT}

Objective We previously reported early-onset atrial fibrillation (AF) associated genetic loci among a Korean population. We explored whether the AF-associated single-nucleotide polymorphisms (SNPs) selected from the Genome-Wide Association Study (GWAS) of an external large cohort has a prediction power for AF in Korean population through a convolutional neural network (CNN). Methods This study included 6358 subjects (872 cases, 5486 controls) from the Korean population GWAS data. We extracted the lists of SNPs at each $p$ value threshold of the association statistics from three different previously reported ethnical-specific GWASs. The Korean GWAS data were divided into training (64\%), validation (16\%) and test (20\%) sets, and a stratified K-fold cross-validation was performed and repeated five times after data shuffling. Results The CNN-GWAS predictive power for AF had an area under the curve (AUC) of $0.78 \pm 0.01$ based on the Japanese GWAS, AUC of $0.79 \pm 0.01$ based on the European GWAS, and AUC of $0.82 \pm 0.01$ based on the multiethnic GWAS, respectively. Gradient-weighted class activation mapping assigned high saliency scores for AF associated SNPs, and the PITX2 obtained the highest saliency score. The CNN-GWAS did not show AF prediction power by SNPs with non-significant $p$ value subset (AUC $0.56 \pm 0.01$ ) despite larger numbers of SNPs. The CNNGWAS had no prediction power for odd-even registration numbers (AUC 0.51 \pm 0.01 ).

Conclusions AF can be predicted by genetic information alone with moderate accuracy. The CNN-GWAS can be a robust and useful tool for detecting polygenic diseases by capturing the cumulative effects and genetic interactions of moderately associated but statistically significant SNPS. Trial registration number NCT02138695.

\section{INTRODUCTION}

Atrial fibrillation (AF) is a major cardiovascular disease with a prevalence of $1.6 \%$ in the total population and is the cause of $20 \%-25 \%$ of ischaemic strokes and about $30 \%$ of heart failure. ${ }^{1} \mathrm{AF}$ is a chronic degenerative disease that progresses from a paroxysmal to persistent type, long-standing persistent and permanent $\mathrm{AF}^{2}$ As more than $50 \%$

\section{Key questions}

What is already known about this subject?

- Atrial fibrillation (AF) is known to be a heritable disease, and multiple genetic loci associated with $\mathrm{AF}$ have been reported by genome-wide association study (GWAS) studies.

What does this study add?

- The collaborative method incorporating a convolutional neural network (CNN) and GWAS could classify the AF vs non-AF with genetic information alone.

- CNN-GWAS with explainable artificial intelligence technique provides a new perspective for GWAS by identifying the positive and negative interactions of each single-nucleotide polymorphism (SNP).

How might this impact on clinical practice?

- CNN-GWAS can be a robust method to predict AF patients by highlighting the cumulative effects and genetic interactions of moderately associated, but statistically significant SNPs. Further studies of comparison and validation with other predictive models are needed to standardised testing.

of AF occurs asymptomatically, early-stage low burden paroxysmal AF is difficult to diagnose by a single examination with an ECG. Moreover, after progressing to persistent AF, rhythm control becomes more difficult than in the paroxysmal AF stage, and the recurrence rate is significantly increased. ${ }^{2}$ Therefore, it is practical to prevent $\mathrm{AF}$ progression or its related complications by an early diagnosis or predicting the occurrence of AF. AF is known to be a heritable disease, and the risk of $\mathrm{AF}$ increases by more than $40 \%$ if a parent or sibling has $\mathrm{AF}^{4}$ As the GenomeWide Association Study (GWAS) has become popular in research, multiple genetic loci related to AF have been reported. ${ }^{5}$ However, it is difficult to find a rare variant gene, and the contribution of genes with intermediate specificity can be neglected because of the 
high specificity of the GWAS. ${ }^{6}$ In addition, the genomewide analysis computes a large amount of genetic information using complex statistical techniques, and therefore, a long and complicated analytic process by a population genetics expert is essential. Because of these technical limitations, the research on the convergence of genetic and clinical information has largely been conducted by multicentre consortiums and serves as a hurdle to the consistent use of the GWAS data in clinical medicine. ${ }^{7}$ As artificial intelligence (AI) research has become more common and popular, the convolutional neural network (CNN) analysis of large-scale genetic data is expected to be faster, more efficient and accurate, but difficulties in interpreting the results still exist. ${ }^{8}$ In this study, we applied CNN and gradient-weighted class activation mapping (Grad-CAM) ${ }^{9}$ to the GWAS analysis to evaluate the effectiveness and accuracy of the method in the prediction power of AF using genomic data. After categorising AF associated single-nucleotide polymorphisms (SNPs) based on the $\mathrm{p}$ values taken from previously reported GWAS summary statistics (Japanese, ${ }^{10}$ European $^{11}$ and multiethnic ${ }^{12}$ studies) independent of the Korean GWAS dataset, we conducted a CNN-GWAS after SNPs encoding with a minor allele. We evaluated the prediction power of the CNN-GWAS and verified it in four different ways including Grad-CAM. The purpose of this study was to investigate the potential of $\mathrm{AI}$ as a tool for the clinical use in the early diagnosis and risk prediction by using genetic information. We also compared AI selected genomes and the early-onset AF associated genetic loci published in our group based on the same GWAS cohort database. ${ }^{5}$

\section{METHODS}

\section{Study design and subjects}

This study protocol adhered to the principles of the Declaration of Helsinki. We included 6358 subjects from four independent cohorts and their GWAS data (figure 1). The case group consisted of 872 patients with early-onset $\mathrm{AF}$ ( $<60$ years old), who underwent AF catheter ablation and had GWAS data available, recruited from the Yonsei AF ablation cohort $(\mathrm{n}=672)$ and Korean AF Network $(n=200$, figure 1).A detailed description is available in the online supplemental material.

\section{Genotyping}

Samples in the genetic dataset were used in our previously published early-onset AF GWAS. ${ }^{5}$ All subjects were extracted the genomic DNA from peripheral blood monocytes by standard procedures and genotyped by the Affymetrix Genome-Wide Human SNP Array V.6.0 chip (Affymetrix, Santa Clara, California, USA). A detailed description is available in online supplemental material.

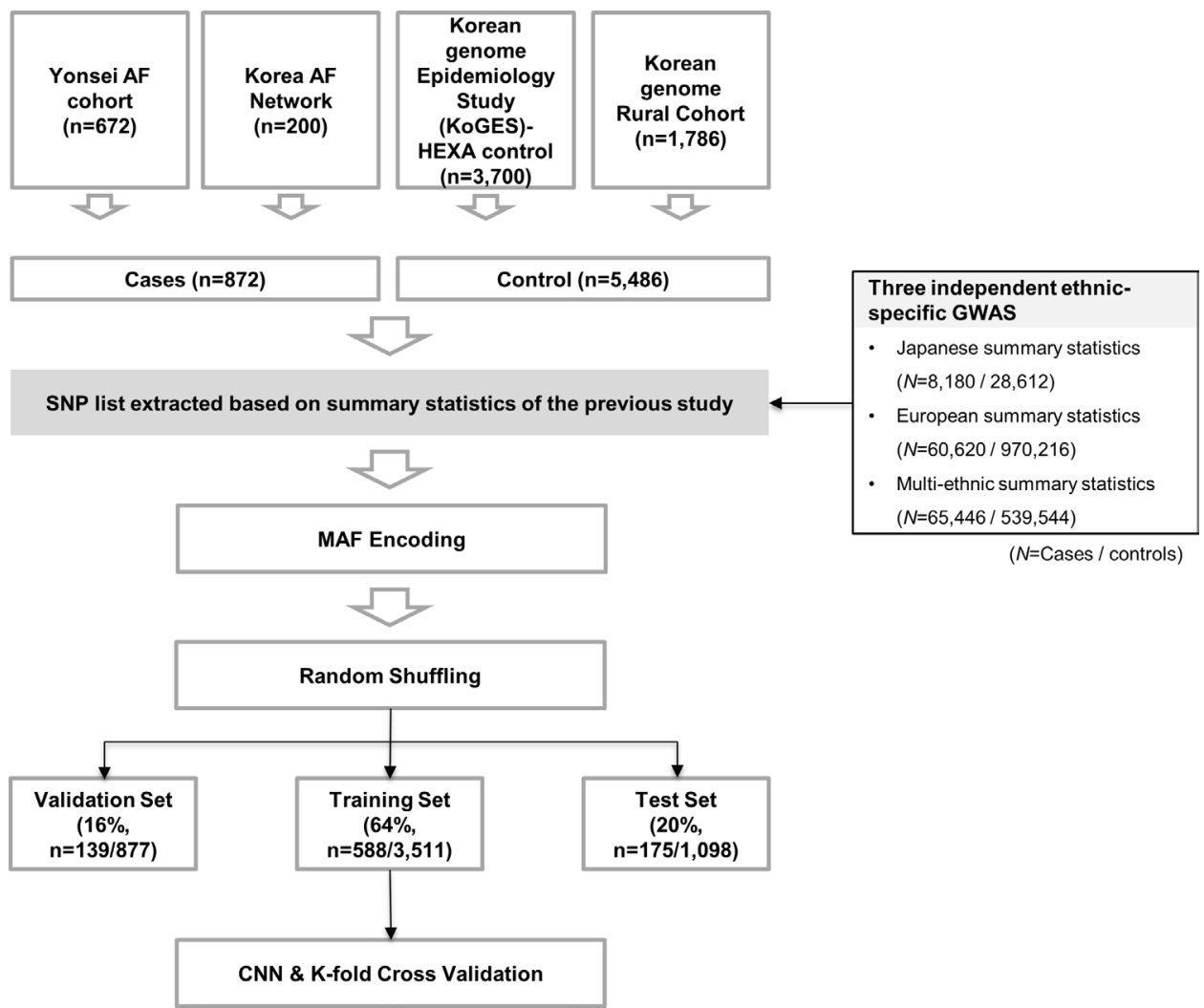

Figure 1 Study flow chart showing the process of the CNN-GWAS, including the AF data set. AF, atrial fibrillation; CNN, convolutional neural network; GWAS, Genome-Wide Association Study; HEXA, health examinee; KoGES, korea genome epidemiology study; MAF, minor allele frequency; SNP, single-nucleotide polymorphism. 


\section{Preprocessing with sampling based on the previously} published GWAS

Too many inputs can cause overfitting, ${ }^{13}$ so we needed the feature selection to remove unnecessary SNPs. Therefore, we preselected from the previously published external GWAS (Japanese, ${ }^{10}$ European $^{11}$ and multiethnic ${ }^{12}$ population cohorts) to ensure reliability and independence (online supplemental table 1). Our total number of SNPs was 531 766, and the numbers of common SNPs mapped to our SNPs were 471462 for Japanese, 530847 for
European and 528039 for multiethnic cohorts, respectively. The set of variants reaching each threshold, ranging from a genome-wide significance level of a $\mathrm{p}<5.0 \times 10^{-8}$ to $\mathrm{p}<0.001$, was considered as a feature selection prior to model training (figure 2A).

\section{Minor allele encoding}

For machine learning (ML), we coded each SNP with homozygous aa 2, heterozygous Aa 1, and AA 0 for the minor allele as an additive model. The missing
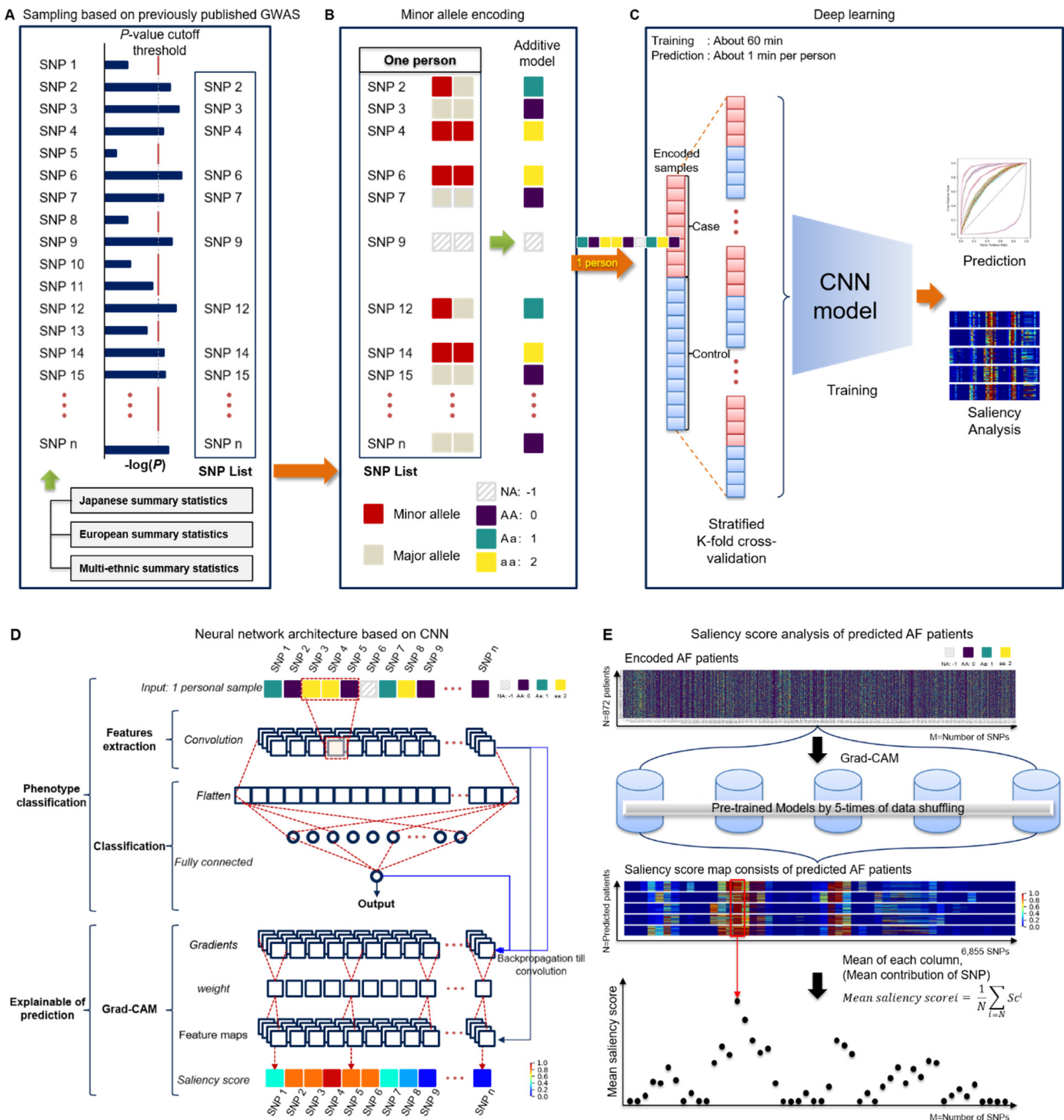

Figure 2 Overview of the CNN-GWAS based framework. (A) A previously reported GWAS-based sampling extracted a set of SNPs according to the $p$ value cut-off. (B) Digitisation encoding for heterozygous or homozygous by minor alleles. (C) Process of the CNN-based neural network model prediction and analysis. (D) Neural network architecture based on the CNN.

(F) Saliency score analysis of the predicted AF patients. The same AF patients $(n=872)$ were used for the quantitative evaluation of the five-time pretrained models using different samples. AF, atrial fibrillation; CNN, convolutional neural network; Grad-CAM, gradient-weighted class activation mapping; GWAS, Genome-Wide Association Study; SNP, single-nucleotide polymorphism. 
genotypes were represented as $-1 .{ }^{14}$ That is, the genotype of each locus was set as the input $X_{m n} \in\{-1,0,1,2\}$ for neurons, where $\mathrm{m}$ is the index in the sample $(1 \leq \mathrm{m} \leq \mathrm{M}, \mathrm{M}=$ the number of samples $)$ and $\mathrm{n}$ is the n-th SNP of the m-th sample (figure $2 B$ ).

\section{Network model design}

We developed a CNN-based model of a hierarchical network so that it can be classified by a locus associated with AF (figure 2A-C). The application of CNN was possible because it can be controlled with imagelike properties in that SNPs are arranged on the same physical base pair. Our network model consisted of two hidden layers. The dimension of the input is the [the number of SNP $\times 1]$. The first layer consisted of a convolutional layer for a feature extract at each SNP level, while the second layer combined into a fully connected layer to perform classification by the associated pattern with the phenotype of AF. The full network is shown in figure 2D. A detailed description is available in online supplemental material.

\section{CNN-GWAS model training}

Early stop and drop-out methods were used to avoid overfitting. Further description is available in online supplemental material.

\section{CNN-GWAS verification}

To verify our model, four validation processes were conducted. First, we repeated the training, validation and test processes five times to demonstrate the reproducibility of the AF prediction and each sample was randomly constructed. Second, to examine whether SNPs of statistically non-significant $\mathrm{p}$ values by a logistic regression did not really affect the AF prediction, an SNP list was constructed and verified based on a $\mathrm{p} \geq 0.99$. Third, in order to identify that there was no predictive power for a phenotype without heritability (here are odd-even registration numbers) other than $\mathrm{AF}$, the validity was verified by replacing the AF label with an odd-even registration number. Fourth, the saliency score of each SNP for AF prediction was analysed in all AF patients $(\mathrm{n}=872)$ using a model of best-performance among the model (figure 2E). A Grad-CAM was applied to calculate the contribution score of each SNP for the AF prediction of the individual. Fifth, to identify whether the issue by class imbalance affected the AF prediction, we conducted a propensity-score matching study.

Further description is available in online supplemental material.

\section{Derivation of polygenic risk score}

To verify the robustness of CNN-GWAS in determining the $\mathrm{AF}$ risk, we evaluated the polygenic risk score (PRS), which is a conventional quantitative metric for the genetic risk. ${ }^{15}$ The PRS was calculated using PLINK software from the same summary statistics as CNN-GWAS and additional criteria for PRS were as follows: removal of SNPs with $r^{2}>0.1$ for linkage disequilibrium-based clumping within $250 \mathrm{~kb}$ range of the index SNP.

\section{Model performance evaluation and statistical analyses}

The data set consisted of mutually exclusive samples with training $(64 \%)$, validation $(16 \%)$ and test $(20 \%)$ sets, each set was selected at random and directly proportional to the number of cases/controls in the population. The final output probability 0 to 1 of a model designed as a binary classifier was evaluated by the phenotype label $\mathrm{Y}=$ (control: 0 or AF patient: 1). The evaluation metrics used the area under the curve (AUC), sensitivity, specificity, positive predictive value, negative predictive value, Gini coefficient, ${ }^{16} \log$-loss and mean square error (MSE). The statistical analyses were performed using R (V.3.6.2) and PLINK software (V.1.9). We also implemented and evaluated the conventional ML methods to compare with the CNN models. We used Bayesian neural network, ${ }^{17}$ Lasso, Ridge and logistic regression to consider the classification problem, and this was developed with a Tensorflow backend. For the Bayesian neural network, the Monte Carlo drop-out rate of 0.5 was applied.

\section{Patient and public involvement}

Patients and/or the public were not involved in the design, or conduct, or reporting, or dissemination plans of this research.

\section{RESULTS \\ Baseline characteristics}

Table 1 summarises the characteristics of the case and control groups in four different cohorts. In 872 $\mathrm{AF}$ patients who underwent $\mathrm{AF}$ catheter ablation, 581 patients $(66.6 \%)$ had paroxysmal AF. The mean age was significantly lower $(50.4 \pm 7.9$ years old vs $55.6 \pm 8.6$ years old, $\mathrm{p}<0.001)$ and the proportion of males was significantly higher $(80.5 \%$ vs $45.5 \%, \mathrm{p}<0.001)$ in the case group than in the control group.

\section{CNN-GWAS prediction model and performance}

The model training time was about $60 \mathrm{~min}$ to learn and the time required to predict the $\mathrm{AF}$ risk of an individual was approximately $1 \mathrm{~min}$ (figure 2C). The training, validation and test set consisted of randomly selected samples, and all tests were repeated five times. Table 2 shows the mean performance results for the $\mathrm{AF}$ predictions in the test sets. The AUC values were $0.78 \pm 0.01$ for Japanese at $\mathrm{p}<0.001,0.79 \pm 0.01$ for European, and $0.82 \pm 0.01$ for multiethnic cohorts at a $\mathrm{p}<1.0 \times 10^{-5}$, respectively (figure $3 \mathrm{~A}-\mathrm{C}$ ). The highest AUC values in each independent cohort are summarised in online supplemental table 3 . The receiver operating characteristic (ROC) curve of the validation set is shown in online supplemental figure 2. In addition, there were no significant differences in comparison of the Bayesian neural network, Lasso and Ridge, but logistic regression showed remarkably less predictive power (figure 4). 
Table 1 Characteristics of the GWAS dataset subjects

\begin{tabular}{|c|c|c|c|c|c|c|}
\hline \multirow[b]{2}{*}{$\begin{array}{l}\text { Baseline } \\
\text { characteristics }\end{array}$} & \multicolumn{2}{|c|}{ Combined } & \multicolumn{2}{|l|}{ Case } & \multicolumn{2}{|l|}{ Control } \\
\hline & $\begin{array}{l}\text { Case } \\
(\mathrm{N}=872)\end{array}$ & $\begin{array}{l}\text { Control } \\
(\mathrm{N}=5486)\end{array}$ & $\begin{array}{l}\text { Yonsei AF } \\
\text { cohort } \\
(\mathrm{N}=672)\end{array}$ & $\begin{array}{l}\text { Korean AF } \\
\text { network } \\
\text { ( } \mathrm{N}=200)\end{array}$ & $\begin{array}{l}\text { KoGES-HEXA } \\
\text { cohort } \\
(\mathrm{N}=3700)\end{array}$ & $\begin{array}{l}\text { Korean genomic } \\
\text { rural cohort } \\
(\mathrm{N}=1786)\end{array}$ \\
\hline Age, year & $50.4 \pm 7.9$ & $55.6 \pm 8.6^{\star}$ & $50.5 \pm 7.8$ & $50.1 \pm 8.2$ & $53.1 \pm 8.3$ & $60.7 \pm 6.6$ \\
\hline Male sex, \% & $702(80.5)$ & $2495(45.5)^{*}$ & $546(81.3)$ & $156(78.0)$ & $1649(44.6)$ & $846(47.4)$ \\
\hline PAF, \% & $581(66.6)$ & - & $482(71.7)$ & $99(49.5)$ & - & - \\
\hline Body mass index, $\mathrm{kg} / \mathrm{m}^{2}$ & $25.0 \pm 3.0$ & $24.2 \pm 3.1^{*}$ & $25.1 \pm 3.0$ & $24.8 \pm 2.8$ & $24.0 \pm 2.9$ & $24.7 \pm 3.3$ \\
\hline Hypertension, \% & $303(34.7)$ & $1278(23.3)^{*}$ & $237(35.3)$ & $66(33.0)$ & $691(18.7)$ & $587(32.9)$ \\
\hline Diabetes, \% & $66(7.6)$ & $899(16.4)^{\star}$ & $51(7.6)$ & $15(7.5)$ & $249(6.7)$ & $650(36.4)$ \\
\hline Coronary artery disease, $\%$ & $77(8.8)$ & $149(2.7)^{*}$ & $56(8.3)$ & $21(10.5)$ & $106(2.9)$ & $43(2.4)$ \\
\hline Stroke, \% & $54(6.2)$ & $115(2.1)^{\star}$ & $47(7.0)$ & $7(3.5)$ & $56(1.5)$ & $59(3.3)$ \\
\hline
\end{tabular}

Data are shown as the mean \pm SD or $\mathrm{n}(\%)$.

${ }^{\star} \mathrm{P}<0.05$.

AF, atrial fibrillation; GWAS, Genome-Wide Association Study; HEXA, health examinee; KoGES, korea genome epidemiology study; PAF, paroxysmal atrial fibrillation.

\section{Model validation with non-significant genomes}

To confirm the validity of the $p$ value cut-off for SNP selection, we conversely evaluated the trained model by selecting SNPs with no statistical association. The SNPs without statistically significant association with AF (cutoff $\mathrm{p} \geq 0.99$ ) were selected in each ethnic-specific GWAS (4221 SNPs in Japanese, 4699 SNPs in European and 4965 SNPs in multiethnic GWAS). Results using these statistically non-significant associated SNPs showed a poor predictive power for AF (AUC 0.56, figure 3A-C). The AF prediction performance estimated by the sensitivity, or specificity, or Gini coefficient were consistently very low (table 2 ).

\section{Model validation by odd-even registration numbers}

To evaluate the robustness of the CNN-GWAS model, we tested whether the AF associated SNPs could predict odd or even registration numbers of the

\begin{tabular}{|c|c|c|c|c|c|c|c|c|c|c|}
\hline Population type & $P$ value & SNPs & AUC & Sens & Spec & PPV & NPV & Gini & Log-loss & MSE \\
\hline \multirow[t]{6}{*}{ Japanese } & $<0.001$ & 2211 & $0.78 \pm 0.01$ & $0.73 \pm 0.05$ & $0.72 \pm 0.04$ & $0.29 \pm 0.02$ & $0.94 \pm 0.01$ & $0.57 \pm 0.02$ & $1.06 \pm 0.80$ & $0.18 \pm 0.14$ \\
\hline & $<1.0 \times 10^{-4}$ & 587 & $0.77 \pm 0.01$ & $0.71 \pm 0.01$ & $0.71 \pm 0.02$ & $0.28 \pm 0.01$ & $0.94 \pm 0.00$ & $0.54 \pm 0.02$ & $0.73 \pm 0.07$ & $0.14 \pm 0.01$ \\
\hline & $<1.0 \times 10^{-5}$ & 262 & $0.75 \pm 0.01$ & $0.70 \pm 0.01$ & $0.69 \pm 0.03$ & $0.26 \pm 0.02$ & $0.94 \pm 0.00$ & $0.50 \pm 0.02$ & $0.73 \pm 0.11$ & $0.18 \pm 0.02$ \\
\hline & $<1.0 \times 10^{-6}$ & 153 & $0.77 \pm 0.01$ & $0.72 \pm 0.03$ & $0.69 \pm 0.04$ & $0.27 \pm 0.02$ & $0.94 \pm 0.00$ & $0.54 \pm 0.01$ & $0.64 \pm 0.03$ & $0.18 \pm 0.01$ \\
\hline & $<5.0 \times 10^{-8}$ & 91 & $0.75 \pm 0.01$ & $0.68 \pm 0.01$ & $0.70 \pm 0.03$ & $0.27 \pm 0.01$ & $0.93 \pm 0.00$ & $0.50 \pm 0.02$ & $0.61 \pm 0.01$ & $0.20 \pm 0.00$ \\
\hline & $\geq 0.990$ & 4221 & $0.56 \pm 0.03$ & $0.54 \pm 0.07$ & $0.56 \pm 0.04$ & $0.16 \pm 0.01$ & $0.88 \pm 0.01$ & $0.12 \pm 0.05$ & $0.98 \pm 0.09$ & $0.16 \pm 0.07$ \\
\hline \multirow[t]{6}{*}{ European } & $<0.001$ & 5401 & $0.74 \pm 0.02$ & $0.72 \pm 0.05$ & $0.65 \pm 0.04$ & $0.25 \pm 0.02$ & $0.94 \pm 0.01$ & $0.48 \pm 0.05$ & $5.46 \pm 0.66$ & $0.42 \pm 0.27$ \\
\hline & $<1.0 \times 10^{-4}$ & 2755 & $0.76 \pm 0.02$ & $0.72 \pm 0.04$ & $0.69 \pm 0.04$ & $0.27 \pm 0.02$ & $0.94 \pm 0.01$ & $0.52 \pm 0.04$ & $3.60 \pm 1.62$ & $0.61 \pm 0.28$ \\
\hline & $<1.0 \times 10^{-5}$ & 1704 & $0.79 \pm 0.01$ & $0.74 \pm 0.03$ & $0.72 \pm 0.04$ & $0.30 \pm 0.02$ & $0.95 \pm 0.01$ & $0.59 \pm 0.03$ & $0.63 \pm 0.05$ & $0.12 \pm 0.02$ \\
\hline & $<1.0 \times 10^{-6}$ & 1192 & $0.78 \pm 0.01$ & $0.72 \pm 0.04$ & $0.71 \pm 0.04$ & $0.29 \pm 0.02$ & $0.94 \pm 0.01$ & $0.57 \pm 0.03$ & $0.72 \pm 0.12$ & $0.13 \pm 0.04$ \\
\hline & $<5.0 \times 10^{-8}$ & 814 & $0.78 \pm 0.01$ & $0.71 \pm 0.03$ & $0.72 \pm 0.03$ & $0.29 \pm 0.02$ & $0.94 \pm 0.00$ & $0.56 \pm 0.02$ & $0.68 \pm 0.04$ & $0.13 \pm 0.03$ \\
\hline & $\geq 0.990$ & 4699 & $0.56 \pm 0.05$ & $0.57 \pm 0.03$ & $0.55 \pm 0.08$ & $0.17 \pm 0.02$ & $0.89 \pm 0.01$ & $0.12 \pm 0.10$ & $2.16 \pm 0.73$ & $0.12 \pm 0.09$ \\
\hline \multirow[t]{6}{*}{ Multiethnic } & $<0.001$ & 4732 & $0.77 \pm 0.01$ & $0.72 \pm 0.05$ & $0.70 \pm 0.04$ & $0.27 \pm 0.02$ & $0.94 \pm 0.01$ & $0.54 \pm 0.02$ & $2.39 \pm 1.61$ & $0.29 \pm 0.34$ \\
\hline & $<1.0 \times 10^{-4}$ & 2372 & $0.79 \pm 0.01$ & $0.72 \pm 0.03$ & $0.73 \pm 0.04$ & $0.30 \pm 0.02$ & $0.94 \pm 0.00$ & $0.58 \pm 0.03$ & $1.19 \pm 0.72$ & $0.13 \pm 0.10$ \\
\hline & $<1.0 \times 10^{-5}$ & 1540 & $0.82 \pm 0.01$ & $0.74 \pm 0.04$ & $0.76 \pm 0.03$ & $0.33 \pm 0.01$ & $0.95 \pm 0.01$ & $0.63 \pm 0.02$ & $0.61 \pm 0.07$ & $0.12 \pm 0.04$ \\
\hline & $<1.0 \times 10^{-6}$ & 1037 & $0.78 \pm 0.02$ & $0.72 \pm 0.03$ & $0.70 \pm 0.04$ & $0.28 \pm 0.02$ & $0.94 \pm 0.01$ & $0.56 \pm 0.04$ & $0.81 \pm 0.16$ & $0.13 \pm 0.01$ \\
\hline & $<5.0 \times 10^{-8}$ & 723 & $0.79 \pm 0.01$ & $0.74 \pm 0.03$ & $0.71 \pm 0.03$ & $0.29 \pm 0.02$ & $0.95 \pm 0.00$ & $0.58 \pm 0.03$ & $0.75 \pm 0.02$ & $0.15 \pm 0.03$ \\
\hline & $\geq 0.990$ & 4965 & $0.56 \pm 0.01$ & $0.56 \pm 0.02$ & $0.56 \pm 0.04$ & $0.17 \pm 0.01$ & $0.89 \pm 0.01$ & $0.12 \pm 0.03$ & $0.98 \pm 0.09$ & $0.11 \pm 0.07$ \\
\hline
\end{tabular}

Data are shown as the mean \pm SD.

The best model of each ethnicity is shown in bold.

AUC, area under the curve; MSE, mean square error; NPV, negative predictive value; PPV, positive predictive value; Sens, sensitivity; SNPs, singlenucleotide polymorphisms; Spec, specificity. 
A ROC of test-set based on Japanese GWAS

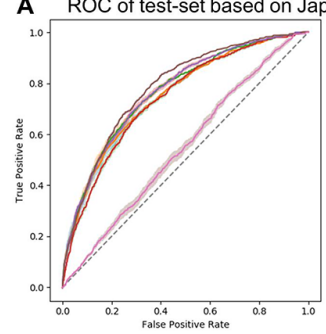

B ROC of test-set based on European GWAS

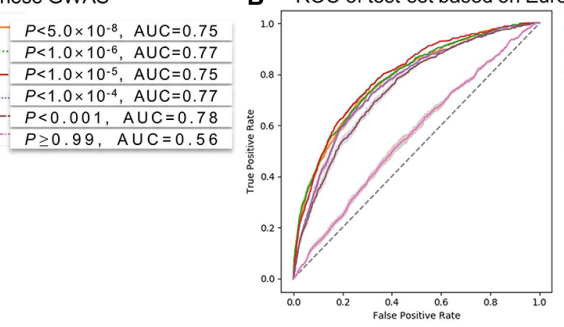

C

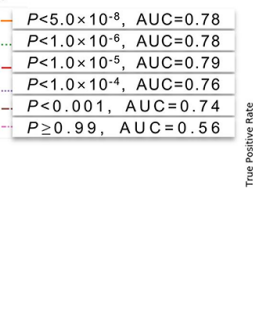

C ROC of test-set based on Multi-ethnic GWAS

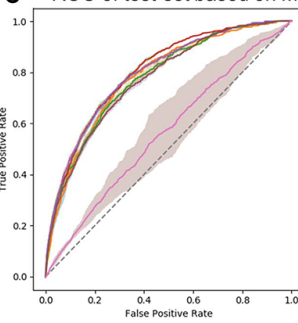

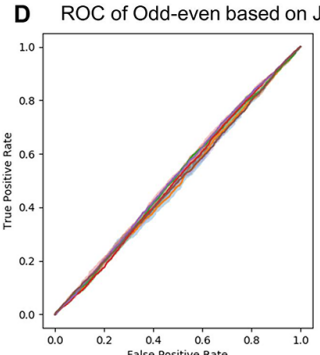
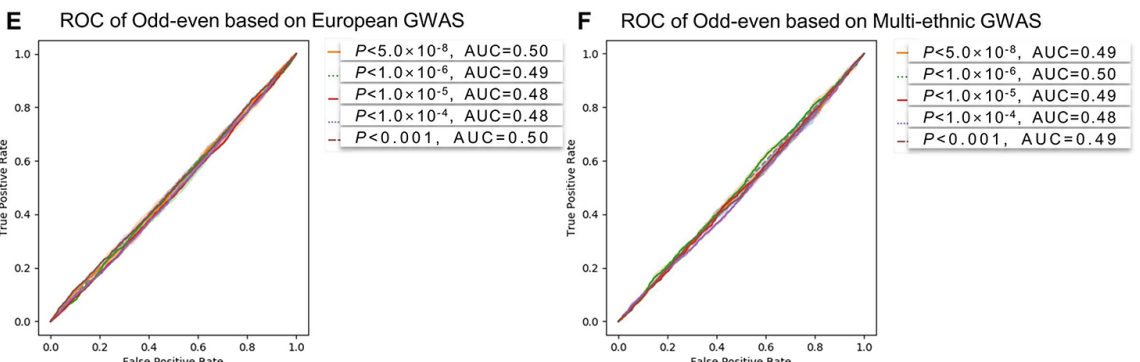

Figure 3 Performance evaluation results. (A-C) The results of the AF prediction ROC curves of the Korean GWAS at each $p$ value cut-off based on the selected SNP set by three different GWAS cohorts' summary statistics (Japanese, European and multiethnic GWAS). P value cutoffs from a $p<0.001$ to $p<5.0 \times 10^{-8}$ were used for the performance evaluation, and a $p \geq 0.99$ was used for the verification of the non-significant SNP list. (D-F) The prediction results for the odd-even registration numbers with the SNP list for the AF prediction ( $p$ value cut-off threshold $p<0.001$ to $p<5.0 \times 10^{-8}$ ). All results were repeated five times, and the shaded area shows the $95 \% \mathrm{Cl}$. AF, atrial fibrillation; AUC, area under the curve; GWAS, Genome-Wide Association Study; ROC, receiver operating characteristic; SNP, single-nucleotide polymorphism.

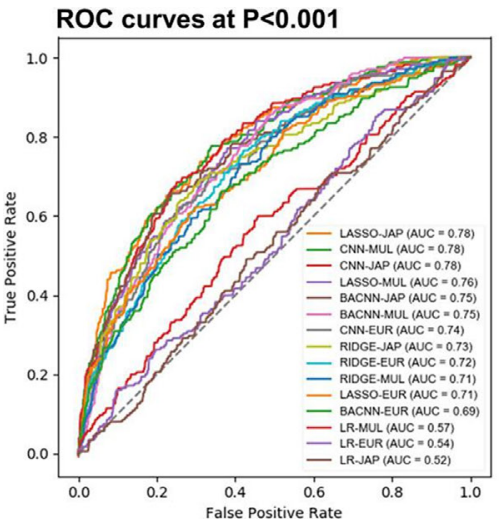

ROC curves at $P<1 \times 10^{-6}$

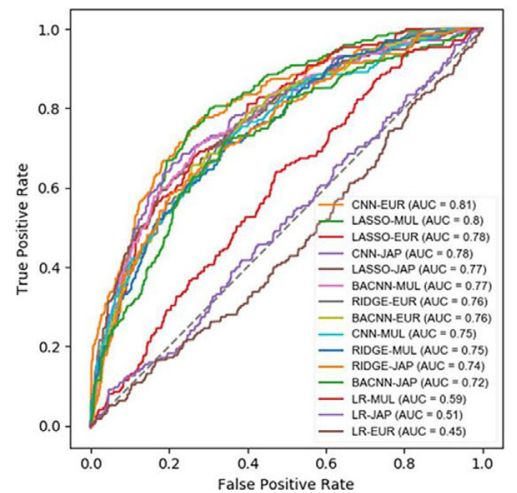

ROC curves at $\mathrm{P}<1 \times 10^{-4}$

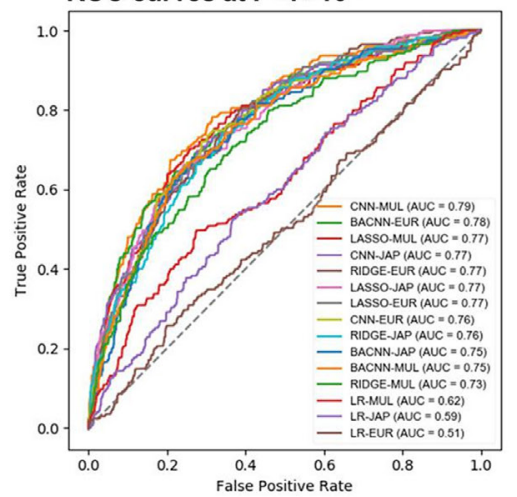

ROC curves at $P<5 \times 10^{-8}$

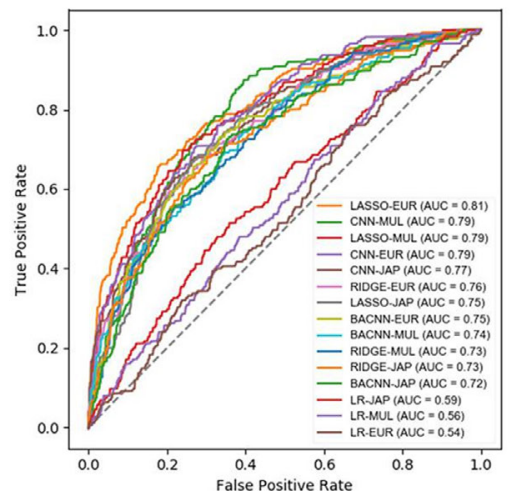

ROC curves at $P<1 \times 10^{-5}$

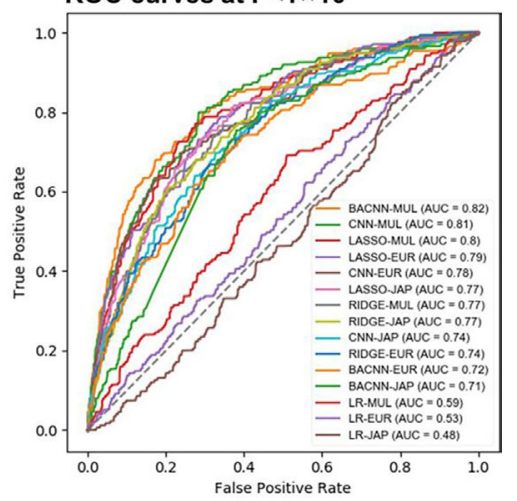

ROC curves at $P>0.99$

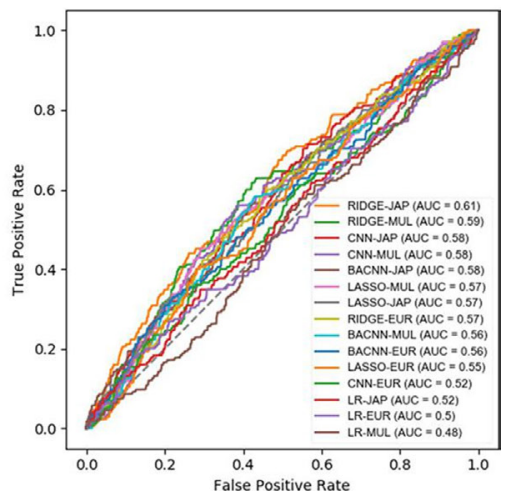

Figure 4 Performance comparison between CNN-GWAS and other machine learning methods. AUC; area under the curve, BACNN; Bayesian approximation convolutional neural network; CNN; convolutional neural network; Eur; European; GWAS, Genome-Wide Association Study; JAP; Japanese; LASSO; least absolute shrinkage and selection operator; LR; logistic regression; MUL; multiethnic; ROC; receiver operating characteristic. 
included population. The numbers of cases and controls separated by odd-even registration numbers were 3189 and 3169 , respectively. The age $(54.8 \pm 8.7$ vs $54.9 \pm 8.8$ years old, $\mathrm{p}=0.799)$ and the proportion of males $(50.0 \%$ vs $50.6 \%, \mathrm{p}=0.633)$ did not significantly differ between the two groups. The ROC curve for odd-even registration numbers did not show any predicted values regardless of the $\mathrm{p}$ value cut-off, and the variation was also small (figure $3 \mathrm{D}-\mathrm{F}$ and online supplemental table 4 ).

\section{Explanation for an AF prediction using the Grad-CAM}

We listed the top $10 \mathrm{SNPs}$ with the highest saliency scores analysed by the Grad-CAM analyses in table 3 . The PITX2, which has been reported as the top first $\mathrm{AF}$ associated gene, exhibited a reproducibly with the

Table 3 Genetic loci with the top 10 mean saliency scores at the high performance $p$ value threshold of each ethnicity

\begin{tabular}{|c|c|c|c|c|c|c|c|c|c|}
\hline Chr. & SNP & Position & Closest gene & $\begin{array}{l}\text { Minor/ } \\
\text { major } \\
\text { allele }\end{array}$ & MAF (\%) & OR & $95 \% \mathrm{Cl}$ & $P$ value & $\begin{array}{l}\text { Mean } \\
\text { saliency } \\
\text { score }\end{array}$ \\
\hline \multicolumn{10}{|c|}{ Based on Japanese GWAS } \\
\hline 4 & rs4611994 & 111711041 & PITX2* & $\mathrm{T} / \mathrm{C}$ & 47.64 & 0.42 & 0.37 to 0.47 & $1.29 \times 10^{-47}$ & 1.000 \\
\hline 4 & rs17042171 & 111708287 & PITX2* & $\mathrm{C} / \mathrm{A}$ & 47.66 & 0.42 & 0.37 to 0.47 & $1.20 \times 10^{-47}$ & 0.711 \\
\hline 4 & rs6843082 & 111718067 & PITX2* & $A / G$ & 27.21 & 0.32 & 0.27 to 0.37 & $8.06 \times 10^{-45}$ & 0.708 \\
\hline 1 & rs3737883 & 203034906 & PPFIA4* & $\mathrm{G} / \mathrm{A}$ & 31.75 & 0.67 & 0.59 to 0.75 & $1.22 \times 10^{-10}$ & 0.498 \\
\hline 4 & rs6852021 & 111744112 & PITX2* & $A / G$ & 49.58 & 0.79 & 0.71 to 0.88 & $1.37 \times 10^{-5}$ & 0.474 \\
\hline 1 & rs11579055 & 203031315 & PPFIA4* & $T / G$ & 32.26 & 0.67 & 0.59 to 0.76 & $1.54 \times 10^{-10}$ & 0.434 \\
\hline 4 & rs723364 & 111724471 & PITX2* & $\mathrm{G} / \mathrm{C}$ & 20.32 & 0.82 & 0.71 to 0.95 & 0.006 & 0.421 \\
\hline 4 & rs17042144 & 111689666 & PITX2* & $\mathrm{C} / \mathrm{T}$ & 42.31 & 2.01 & 1.80 to 2.24 & $1.40 \times 10^{-34}$ & 0.403 \\
\hline 1 & rs66944777 & 203035365 & PPFIA4* & $\mathrm{G} / \mathrm{A}$ & 21.05 & 0.64 & 0.55 to 0.74 & $1.53 \times 10^{-9}$ & 0.386 \\
\hline 20 & rs 11696871 & 62387417 & ZBTB46 & $\mathrm{A} / \mathrm{G}$ & 48.64 & 0.94 & 0.84 to 1.04 & 0.242 & 0.385 \\
\hline \multicolumn{10}{|c|}{ Based on European GWAS } \\
\hline 4 & rs4611994 & 111711041 & PITX2* & $T / C$ & 47.64 & 0.42 & 0.37 to 0.47 & $1.29 \times 10^{-47}$ & 1.000 \\
\hline 4 & rs17042171 & 111708287 & PITX2 & $\mathrm{C} / \mathrm{A}$ & 47.66 & 0.42 & 0.37 to 0.47 & $1.20 \times 10^{-47}$ & 0.740 \\
\hline 4 & rs6843082 & 111718067 & PITX2* & $\mathrm{A} / \mathrm{G}$ & 27.21 & 0.32 & 0.27 to 0.37 & $8.06 \times 10^{-45}$ & 0.716 \\
\hline 1 & rs11579055 & 203031315 & PPFIA4* & $\mathrm{T} / \mathrm{G}$ & 32.26 & 0.67 & 0.59 to 0.76 & $1.54 \times 10^{-10}$ & 0.488 \\
\hline 1 & rs3737883 & 203034906 & PPFIA4* & $\mathrm{G} / \mathrm{A}$ & 31.75 & 0.67 & 0.59 to 0.75 & $1.22 \times 10^{-10}$ & 0.486 \\
\hline 22 & rs464385 & 18571008 & $T U B A B^{\star}$ & $A / G$ & 43.12 & 1.01 & 0.91 to 1.13 & 0.824 & 0.485 \\
\hline 4 & rs 17042144 & 111689666 & PITX2* & $\mathrm{C} / \mathrm{T}$ & 42.31 & 2.01 & 1.80 to 2.24 & $1.40 \times 10^{-34}$ & 0.457 \\
\hline 22 & rs361594 & 18577338 & $T U B A 8^{\star}$ & $T / C$ & 48.19 & 0.91 & 0.82 to 1.02 & 0.105 & 0.440 \\
\hline 1 & rs6694477 & 203035365 & PPFIA4* & $\mathrm{G} / \mathrm{A}$ & 21.05 & 0.64 & 0.55 to 0.74 & $1.53 \times 10^{-9}$ & 0.401 \\
\hline 4 & rs3866838 & 111753815 & PITX2 & $T / C$ & 17.75 & 0.85 & 0.73 to 0.98 & 0.025 & 0.399 \\
\hline \multicolumn{10}{|c|}{ Based on multi-ethnic GWAS } \\
\hline 4 & rs4611994 & 111711041 & PITX2 & $T / C$ & 47.64 & 0.42 & 0.37 to 0.47 & $1.29 \times 10^{-47}$ & 1.000 \\
\hline 4 & rs6843082 & 111718067 & PITX2* & $A / G$ & 27.21 & 0.32 & 0.27 to 0.37 & $8.06 \times 10^{-45}$ & 0.827 \\
\hline 1 & rs3737883 & 203034906 & PPFIA4* & $\mathrm{G} / \mathrm{A}$ & 31.75 & 0.67 & 0.59 to 0.75 & $1.22 \times 10^{-10}$ & 0.634 \\
\hline 4 & rs17042171 & 111708287 & PITX2* & $\mathrm{C} / \mathrm{A}$ & 47.66 & 0.42 & 0.37 to 0.47 & $1.20 \times 10^{-47}$ & 0.540 \\
\hline 4 & rs2067518 & 174612666 & $H A N D 2^{*}$ & $\mathrm{G} / \mathrm{A}$ & 45.85 & 0.73 & 0.65 to 0.81 & $1.38 \times 10^{-8}$ & 0.531 \\
\hline 1 & rs11579055 & 203031315 & PPFIA4* & $T / G$ & 32.26 & 0.67 & 0.59 to 0.76 & $1.54 \times 10^{-10}$ & 0.527 \\
\hline 1 & rs66944777 & 203035365 & PPFIA4* & $\mathrm{G} / \mathrm{A}$ & 21.05 & 0.64 & 0.55 to 0.74 & $1.53 \times 10^{-9}$ & 0.515 \\
\hline 4 & rs 12507756 & 174609772 & $\operatorname{HAND2}^{*}$ & $\mathrm{C} / \mathrm{T}$ & 45.78 & 0.73 & 0.65 to 0.81 & $2.00 \times 10^{-8}$ & 0.459 \\
\hline 1 & rs6689393 & 154426097 & $K C N N 3^{\star}$ & $\mathrm{A} / \mathrm{G}$ & 45.99 & 1.07 & 0.96 to 1.19 & 0.199 & 0.458 \\
\hline 1 & rs535709 & 170120831 & METTL11B & $\mathrm{C} / \mathrm{T}$ & 12.92 & 1.04 & 0.88 to 1.22 & 0.648 & 0.430 \\
\hline
\end{tabular}

${ }^{*}$ Genes are previously proven AF associated loci.

AF, atrial fibrillation; Chr., chromosome; GWAS, Genome-Wide Association Study; MAF, minor allele frequency; SNP, single-nucleotide polymorphism. 
A Manhattan plot of Korean GWAS displaying selected SNPs set by Multi-ethnic GWAS (at $\left.P<1.0 \times 10^{-5}\right)$

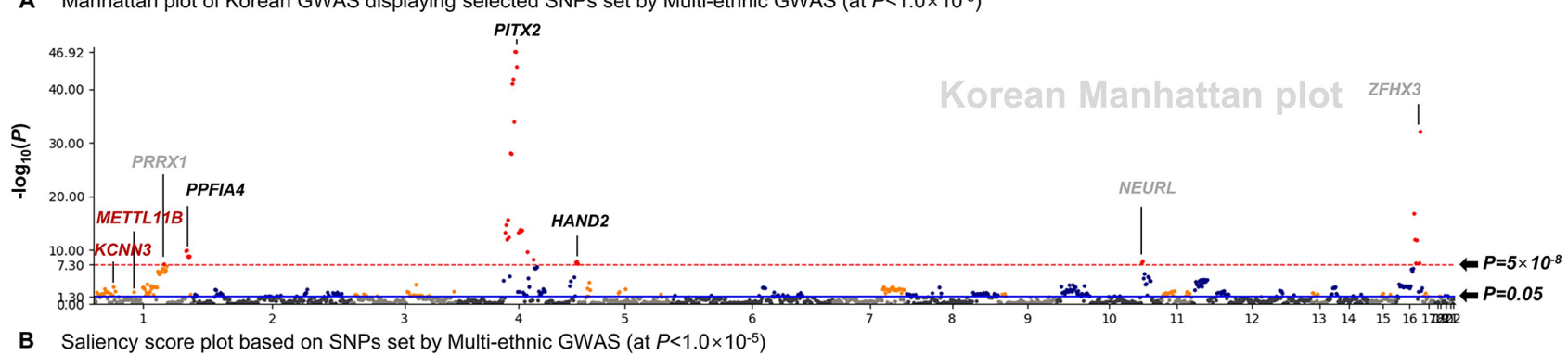

B Saliency score plot based on SNPs set by Multi-ethnic GWAS (at $P<1.0 \times 10^{-5}$ )
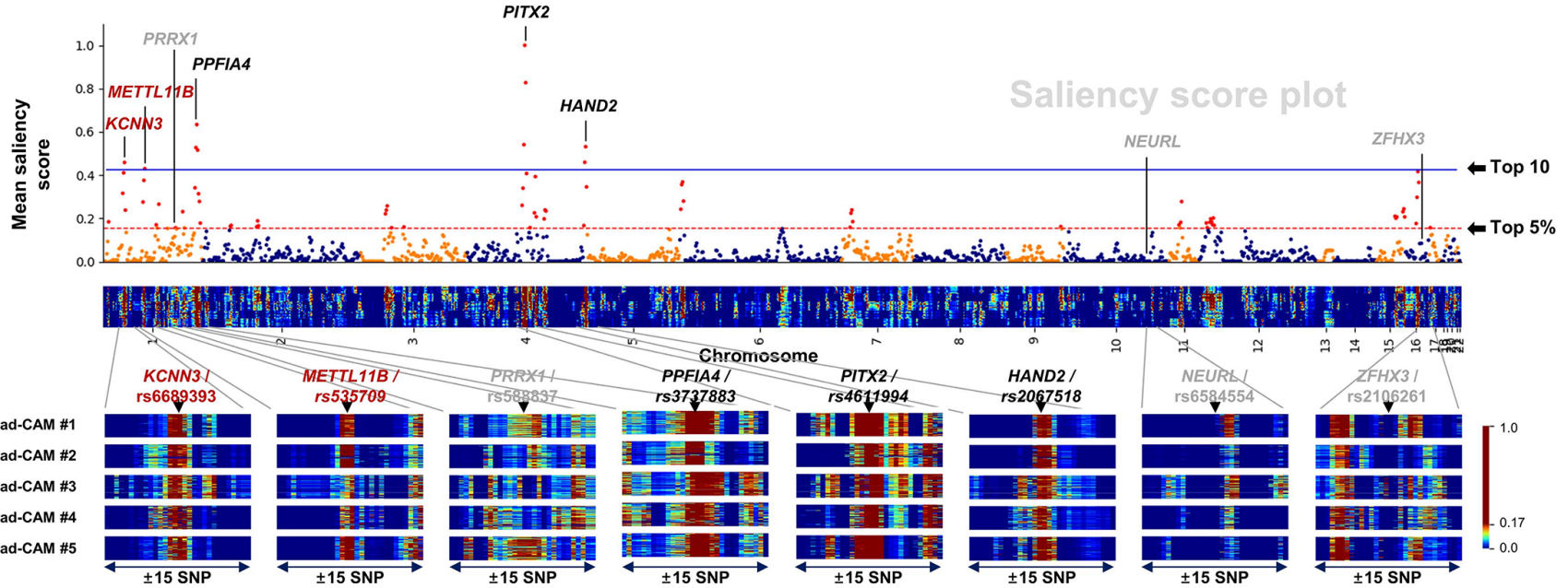

Figure 5 Explanation of the predictive power of the CNN-GWAS for AF. (A) The Manhattan plot of the Korean population GWAS represented by the SNP set selected at a $p<1.0 \times 10^{-5}$ in the multiethnic GWAS. (B) The contribution of each SNP for the AF prediction is represented by a plot (top), which is the mean saliency score for each column of the two-dimensional (2D) saliency score map. The saliency scores of each AF patient are displayed stacked in the 2D saliency score map (below). Those in the grey font were reported to be AF associated SNPs but were not included in the top 10 highest salience scored SNPs. The blue horizontal line stands for the top 10 saliency score levels, and the red dotted horizontal line stands for the top $5 \%$ high saliency score levels. AF, atrial fibrillation; CNN, convolutional neural network; Grad-CAM, gradient-weighted class activation mapping; GWAS, Genome-Wide Association Study; SNP, single-nucleotide polymorphism.

highest saliency scores in all three independent cohorts of different ethnicities. The other proven AF associated SNPs, such as KCNN3, METTL11B, PPFIA4, HAND2 and TUBA8, were also included in the top 10 highest saliency scores. The Pearson correlation coefficient was 0.472 when comparing the Manhattan plot and Saliency score plot, and those displayed the selected SNPs set by the multiethnic GWAS at a $\mathrm{p}<1.0 \times 10^{-5}$ (figure 5). In the Manhattan plot (figure 5A), 15 of 36 significant AF-associated SNPs with a genome-wide significance $\left(\mathrm{p}<5.0 \times 10^{-8}\right)$ were ranked in the top $5 \%$ of the saliency score plot. Conversely, 75 out of 78 SNPs ranked in the top $5 \%$ of the saliency score plot (figure 5B) were previously proven AF associated genetic loci in the multiethnic cohort, of which 54 SNPs were replicated in the Korean GWAS at a $\mathrm{p}<0.05$ level (figure $5 \mathrm{~A}$ ).

\section{CNN-GWAS performance after propensity-score matching}

After 1:1 propensity-score matching, 862 AF patient group and 862 control group were compared with test the AF prediction power of CNN-GWAS (online supplemental table 5). After five repeated analyses with CNN-GWAS, the AUC values were $0.78 \pm 0.01$ for Japanese, $0.78 \pm 0.01$ for European at $\mathrm{p}<5.0 \times 10^{-8}, 0.78 \pm 0.01$ for multiethnic group at $\mathrm{p}<1.0 \times 10^{-5}$, respectively.

\section{Prediction results by PRS}

We evaluated AF prediction power based on the PRS. The numbers of SNPs for PRS calculation by the $p$ value cut-off are displayed in online supplemental table 6 . The AUC values of Japanese and multiethnic groups were 0.82 and 0.83 , respectively, at $\mathrm{p}<0.001$. There were no significant differences compared with the predictive power of CNN-GWAS. However, the AUC value of European was 0.72 at $\mathrm{p}<1.0 \times 10^{-6}$, which showed decreased predictive power compared with other models.

\section{DISCUSSION \\ Main findings}

In this study, we explored whether a collaborative method of the CNN and GWAS was feasible in predicting the risk of AF based on the genetic data of a large population. The CNN-GWAS model achieved a reasonably acceptable AF prediction power (AUC 0.74 0.82) in the Korean population by utilising moderate AF-associated SNPs proven in three independent cohorts with different ethnicities. 
We verified the CNN-GWAS model by randomly shuffling the dataset five times, demonstrating no AF predictive power using SNPs with non-significant $P$-value subsets and no predictive powers for odd and even cohort registration numbers using genetic information. The predictive model of CNN-GWAS showed a stable predictive power compared with PRS even when GWAS summary statistics derived from other ancestry cohorts are applied to different ethnic cohorts. We also confirmed the high impact of pre-reported $\mathrm{AF}$ associated genetic loci on the AF prediction power in the CNN-GWAS model that were trained in the right direction by the Grad-CAM method. The CNN-GWAS algorithms capture the cumulative effects and genetic interactions of less significant or undiscovered SNPs that determine the manifestation of the AF phenotype.

\section{Emerging roles of the CNN in clinical cardiology}

The use of AI, which enables a fast, sophisticated diagnosis, treatment and improved patient care workflow, and precision medical care, is increasing in clinical practice. $\mathrm{AI}$ is particularly useful for analysing data-rich technologybased objectives, such as omics, mobile device biometrics and electronic health records to obtain clinically useful information. ${ }^{18}$ The high predictive power of $\mathrm{AI}$ is also useful for cardiovascular disease, a slowly progressive disease with multifactorial pathophysiology and cardiac arrhythmia disease, which is difficult to predict, occurs suddenly and causes various complications. ${ }^{19} \mathrm{AI}$ has been variously tested for the diagnostic purposes of cardiac diseases,${ }^{20}$ and its high prognostic prediction power in cardiac imaging and electrocardiograms has already been verified. ${ }^{21}$ In this study, in combination with the GWAS data, AI demonstrated very high predictability of the common cardiac arrhythmia, AF, without including the clinical characteristics, personal habits or environmental factors. This further supports the evidence that $\mathrm{AF}$ is a heritable disease strongly affected by genetic factors. ${ }^{4522}$

\section{Implications of CNN-GWAS-based precision medicine}

In this study, we used AI algorithms of supervised learning techniques and the $\mathrm{CNN}$, which is a deep learning method. Because a large well-curated clinical dataset is essential to properly train the deep learning, ${ }^{23}$ well quality-controlled genetic information has advantages over other clinical dataset. The advantages of deep learning are easy image recognition, no working memory limitations and its use with both supervised and nonsupervised learning. ${ }^{19}$ On the other hand, the weaknesses of deep learning including the CNN are the possibility of overfitting and the error of learning when providing a biased training dataset. These two problems can be overcome by increasing the sample size of the training dataset or decreasing the number of hidden layers. ${ }^{24}$ The K-fold cross-validation is reported to be more accurate than the traditional split-sample approach. ${ }^{25}$ In this study, we used a single convolution and a fully connected layer and the K-fold cross-validation to evaluate over a half million genomic data of 6358 subjects. The output of the CNNGWAS was verified in four different ways. Moreover, the black-box region, which is a chronic problem in the $\mathrm{CNN}$ analyses, was partially analysed by the Grad-CAM method, and AI calculations assigned high contribution scores to prediscovered $\mathrm{AF}$ associated genetic loci, especially PITX2. It is expected that the prudent monitoring of one $\mathrm{AI}$ algorithm by another $\mathrm{AI}$ algorithm will be used in the future.

\section{Study limitations}

There are several potential limitations to our study. First, the results of this study cannot be generalised due to the nature of AI, which is greatly influenced by the training dataset. Second, this study included a highly selected group of patients (60 years old and younger) who were referred for $\mathrm{AF}$ ablation. This select patient population represents symptomatic antiarrhythmic drug-resistant early-onset AF. Third, the outcome of this study based on the Korean AF cohort data may not be generalised to other cohorts with different ethnicities and races. Fourth, the reason why a $\mathrm{p} \geq 0.99$ was used for the SNP analysis of the non-significant $p$ values used for validation of this study is due to the limitation of the computing power when it is executed at a $p \geq 0.05$. Fifth, the sample size of this study is relatively small compared with other largescale GWAS studies. However, it satisfies the research purpose of evaluating the reproducibility of AF-associated SNPs after CNN application in the same patient group as our previous study proven by conventional statistical methods.

\section{CONCLUSIONS}

In summary, the CNN-GWAS algorithm can be used to predict the $\mathrm{AF}$, but comparison and verification with other models will be further warranted. The CNN-GWAS algorithms capture the cumulative effects and genetic interactions of moderately associated but statistically significant genes that determine the manifestation of the $\mathrm{AF}$ phenotype. AF can be predicted by genetic information alone with moderate accuracy.

Acknowledgements This study was conducted with bioresources from National Biobank of Korea, the Center for Disease Control and Prevention, Republic of Korea. (KBN-2016-005). We would like to thank Mr John Martin for his linguistic assistance.

Contributors 0 -SK and H-NP designed the study, analysed and interpreted the data, and wrote the manuscript. MH and T-HK analysed and interpreted the data. $\mathrm{MH}$ and $\mathrm{IH}$ genotyped the data. JS, E-KC, HEL, HTY, J-SU, BJ, SO, M-HL, Y-HK and $\mathrm{H}-\mathrm{NP}$ contributed to acquiring patients' genetic data. H-NP is the guarantor who accepts full responsibility for the finished work and/or the conduct of the study, had access to the data, and controlled the decision to publish.

Funding This work was supported by grants (HI19C0114 to H-NP) and (HI21C0011 to H-NP) from the Ministry of Health and Welfare, grants (NRF-2020R1A2B01001695 to H-NP) and (NRF-2019R111A1A01041440 to MH) from the Basic Science Research Programme through the National Research Foundation of Korea (NRF) funded by the Ministry of Education.

Competing interests None declared.

Patient consent for publication Consent obtained directly from patient(s) 
Ethics approval This study was approved by the Institutional Review Board of Yonsei University Health System, the Institutional Review Board of Korea University Cardiovascular Center, the Institutional Review Board of Seoul National University, the Institutional Review Board of Hallym University.

Provenance and peer review Not commissioned; externally peer reviewed.

Data availability statement Data may be obtained from a third party and are not publicly available. Data contain sensitive patient information. Sharing of data is restricted by ethical approvals and the Personal Information Protection Act of the Republic of Korea. Access to data to reproduce results requires the application to and permission from Professor Hui-Nam Pak and The National Biobank of Korea.

Open access This is an open access article distributed in accordance with the Creative Commons Attribution 4.0 Unported (CC BY 4.0) license, which permits others to copy, redistribute, remix, transform and build upon this work for any purpose, provided the original work is properly cited, a link to the licence is given, and indication of whether changes were made. See: https://creativecommons.org/ licenses/by/4.0/.

ORCID iD

Hui-Nam Pak http://orcid.org/0000-0002-3256-3620

\section{REFERENCES}

1 Kim D, Yang P-S, Jang E, et al. 10-Year nationwide trends of the incidence, prevalence, and adverse outcomes of non-valvular atrial fibrillation nationwide health insurance data covering the entire Korean population. Am Heart J 2018;202:20-6.

2 Kirchhof P, Benussi S, Kotecha D, et al. 2016 ESC guidelines for the management of atrial fibrillation developed in collaboration with EACTS. Eur Heart J 2016;37:2893-962.

3 Steg PG, Alam S, Chiang C-E, et al. Symptoms, functional status and quality of life in patients with controlled and uncontrolled atrial fibrillation: data from the RealiseAF cross-sectional international registry. Heart 2012;98:195-201.

4 Lubitz SA, Yin X, Fontes JD, et al. Association between familia atrial fibrillation and risk of new-onset atrial fibrillation. JAMA 2010;304:2263-9.

5 Lee J-Y, Kim T-H, Yang P-S, et al. Korean atrial fibrillation network genome-wide association study for early-onset atrial fibrillation identifies novel susceptibility loci. Eur Heart J 2017;38:2586-94.

6 Choi SH, Weng L-C, Roselli C, et al. Association between titin loss-of-function variants and early-onset atrial fibrillation. JAMA 2018:320:2354-64.

7 Choi E-K, Park JH, Lee J-Y, et al. Korean atrial fibrillation (AF) network: genetic variants for AF do not predict ablation success. $J$ Am Heart Assoc 2015;4:e002046.
8 Bellot P, de Los Campos G, Pérez-Enciso M. Can deep learning improve genomic prediction of complex human traits? Genetics 2018;210:809-19.

9 Selvaraju RR, Cogswell M, Das A. Grad-cam: visual explanations from deep networks via gradient-based localization. Proceedings of the IEEE International Conference on Computer Vision 2017:618-26.

10 Low S-K, Takahashi A, Ebana Y, et al. Identification of six new genetic loci associated with atrial fibrillation in the Japanese population. Nat Genet 2017;49:953-8.

11 Nielsen JB, Thorolfsdottir RB, Fritsche LG, et al. Biobank-driven genomic discovery yields new insight into atrial fibrillation biology. Nat Genet 2018:50:1234-9.

12 Roselli C, Chaffin MD, Weng L-C, et al. Multi-Ethnic genome-wide association study for atrial fibrillation. Nat Genet 2018;50:1225-33.

13 Kohavi R, Sommerfield D. Feature subset selection using the wrapper method: Overfitting and dynamic search space topology. KDD 1995:192-7.

14 Liu Y, Wang D, He F, et al. Phenotype prediction and genome-wide association study using deep Convolutional neural network of soybean. Front Genet 2019:10:1091.

15 Lewis CM, Vassos E. Polygenic risk scores: from research tools to clinical instruments. Genome Med 2020;12:44.

16 Hand DJ, Till RJ. A simple generalisation of the area under the ROC curve for multiple class classification problems. Mach Learn 2001;45:171-86.

17 Gal Y, Ghahramani Z. Dropout as a Bayesian approximation: representing model uncertainty in deep learning. International Conference on Machine Learning: PMLR 2016:1050-9.

18 Muse ED, Barrett PM, Steinhubl SR, et al. Towards a smart medical home. The Lancet 2017;389:358.

19 Dey D, Slomka PJ, Leeson P, et al. Artificial intelligence in cardiovascular imaging: JACC state-of-the-art review. J Am Coll Cardiol 2019;73:1317-35.

20 Betancur J, Commandeur F, Motlagh M, et al. Deep learning for prediction of obstructive disease from fast myocardia perfusion SPECT: a multicenter study. JACC Cardiovasc Imaging 2018:11:1654-63.

21 Motwani M, Dey D, Berman DS, et al. Machine learning for prediction of all-cause mortality in patients with suspected coronary artery disease: a 5-year multicentre prospective registry analysis. Eur Heart J 2017;38:500-7.

22 Arnar DO, Thorvaldsson S, Manolio TA, et al. Familial aggregation of atrial fibrillation in Iceland. Eur Heart J 2006;27:708-12.

23 Johnson KW, Torres Soto J, Glicksberg BS, et al. Artificial intelligence in cardiology. J Am Coll Cardiol 2018;71:2668-79.

24 Duch W, Jankowski N, Maszczyk T. Make it cheap: learning with O (nd) complexity. The 2012 International Joint Conference on Neural Networks (IJCNN): IEEE 2012:1-4.

25 Ellis RP, Mookim PG. K-Fold cross-validation is superior to split sample validation for risk adjustment models. Boston UniversityDepartment of Economics, 2013. 